\title{
Roles of vimentin and 14-3-3 zeta/delta in the inhibitory effects of heparin on PC-3M cell proliferation and B16-F10-luc-G5 cells metastasis
}

\author{
Yan PAN ${ }^{1}$, Li-jun ZHONG ${ }^{2}$, Hong ZHOU ${ }^{1}$, Xin WANG ${ }^{1}$, Kui CHEN ${ }^{1}$, Hao-peng YANG ${ }^{1}$, Yilixiati XIAOKAITI ${ }^{1}$, Aikebaier MAIMAITI ${ }^{1}$, \\ Ling JIANG ${ }^{1}$, Xue-jun $\mathrm{LI}^{1,}$ *

\begin{abstract}
${ }^{1}$ State Key Laboratory of Natural and Biomimetic Drugs, Department of Pharmacology, School of Basic Medical Sciences, Peking University and Institute of System Biomedicine, Peking University, Beijing 100191, China; ${ }^{2}$ Center of Medical Analysis, Peking University, Beijing 100191, China
\end{abstract}

\begin{abstract}
Aim: To investigate the inhibitory effects of heparin on PC-3M cells proliferation in vitro and B16-F10-luc-G5 cells metastasis in Balb/c nude mice and identify the protein expression patterns to elucidate the action mechanism of heparin.

Methods: Human prostate cancer PC-3M cells were incubated with heparin 0.5 to $125 \mu \mathrm{g} / \mathrm{mL}$ for $24 \mathrm{~h}$. The proliferation of PC-3M cells was assessed by MTS assay. BrdU incoporation and Ki67 expression were detected using a high content screening (HCS) assay. The cell cycle and apoptosis of PC-3M cells were tested by flow cytometry. B16-F10-luc-G5 cardinoma cells were injected into the lateral tail vein of 6-week old male Balb/c nude mice and heparin $30 \mathrm{mg} / \mathrm{kg}$ was administered iv $30 \mathrm{~min}$ before and $24 \mathrm{~h}$ after injection. The metasis of B16-F10-luc-G5 cells was detected by bioluminescence assay. Activated partial thromboplastin time (APTT) and hemorheological parameters were measured on d 14 after injection of B16-F10-luc-G5 carcinoma cells in Balb/c mice. The global protein changes in PC-3M cells and frozen lung tissues from mice burdened with B16-F10-luc-G5 cells were determined by 2-dimensional gel electrophoresis and image analysis. The protein expression of vimentin and 14-3-3 zeta/delta was measured by Western blot. The mRNA transcription of vimentin, transforming growth factor (TGF)- $\beta$, E-cadherin, and $\alpha_{v}$-integrin was measured by RT-PCR.

Results: Heparin 25 and $125 \mu \mathrm{g} / \mathrm{mL}$ significantly inhibited the proliferation, arrested the cells in $\mathrm{G}_{1}$ phase, and suppressed BrdU incorporation and Ki67 expression in PC-3M cells compared with the model group. But it had no significant effect on apoptosis of PC-3M cells. Heparin $30 \mathrm{mg} / \mathrm{kg}$ markedly inhibits the metastasis of B16-F10-luc-G5 cells on day 8. Additionally, heparin administration maintained relatively normal red blood hematocrit but had no influence on APTT in nude mice burdened with B16-F10-luc-G5 cells. Thirty of down-regulated protein spots were identified after heparin treatment, many of which are related to tumor development, extracellular signaling, energy metabolism, and cellular proliferation. Vimentin and 14-3-3 zeta/delta were identified in common in PC-3M cells and the lungs of mice bearing B16-F10-luc-G5 carcinoma cells. Heparin 25 and $125 \mu \mathrm{g} / \mathrm{mL}$ decreased the protein expression of vimentin and 14-3-3 zeta/delta and the mRNA expression of $\alpha_{v}$-integrin. Heparin $125 \mu \mathrm{g} / \mathrm{mL}$ decreased vimentin and E-cadherin mRNA transcription while increased TGF- $\beta$ mRNA transcription in the PC-3M cells, but the differences were not significant. Transfection of vimentin-targeted siRNA for $48 \mathrm{~h}$ significantly decreased the BrdU incoporation and Ki67 expression in PC-3M cells.

Conclusion: Heparin inhibited PC-3M cell proliferation in vitro and B16-F10-luc-G5 cells metastasis in nude mice by inhibition of vimentin, 14-3-3 zeta/delta, and $\alpha_{v}$-integrin expression.
\end{abstract}

Keywords: heparin; cell growth; proteome; vimentin; 14-3-3 zeta/delta; $\alpha_{\mathrm{v}}$-integrin

Acta Pharmacologica Sinica (2012) 33: 798-808; doi: 10.1038/aps.2012.42

\section{Introduction}

Despite the progress made over decades of research, cancer remains a major threat to human health with significant morbidity and mortality. In the development of new anti-

\footnotetext{
* To whom correspondence should be addressed.

E-mail xjli@bjmu.edu.cn

Received 2012-02-14 Accepted 2012-03-30
}

tumor treatments, multi-targeting drugs continue to emerge as investigators search for candidates among currently available agents. Drugs with a long history of safe clinical use are especially attractive for testing their efficacy against cancer, which may be outside of the scope of their original application. In addition to its anti-coagulant activity, heparin is known to have suppressive effects on multiple biologic functions, such as hypertension ${ }^{[1]}$, inflammation ${ }^{[2]}$, and cellular proliferation ${ }^{[3]}$. 
Heparin is a polysulfated glycosaminoglycan with a highly negative charge. Two trials (FAMOUS and MALT) previously studied the effect of unfractionated heparin $(\mathrm{UFH})^{[4]}$ or low molecular weight heparin $(\mathrm{LMWH})^{[5]}$ in patients with small cell lung cancer. In both of these studies, a statistically significant survival advantage was observed in patients randomized to chemotherapy plus UFH or LMWH relative to patients who received chemotherapy alone. While several clinical studies strongly support an anti-cancer activity of heparin, especially $\mathrm{LMWH}$, many questions remain unresolved ${ }^{[6]}$. For example, it is still unknown whether and how heparin modulates survival of carcinoma cells, although a number of biological properties have been postulated to explain the effects of heparin on the malignant process.

In this investigation, we addressed the potential inhibitory effects of heparin on growth of human prostate cancer cells (PC-3M) and metastasis of B16-F10-luc-G5 carcinoma cells in $\mathrm{Balb} / \mathrm{c}$ nude mice with single dose and twice injection way and monitoring with living imaging technology. For the spontaneous hemorrhage is the main side effects of heparin, we also evaluated the systemic bleeding parameters of activated partial thromboplastin time (APTT) and hemorheological parameters of whole blood viscosity, elongation index $(\mathrm{EI})$, orientation index $(\mathrm{OI})$ and hematocrit of red blood cells, which are related greatly with tumor metastasis. By using a proteomic approach, we also identified proteins significantly associated with the anti-tumor and anti-metastatic effects of heparin to explore its new molecular mechanisms.

\section{Materials and methods Materials}

DMEM medium, fetal bovine serum (FBS), penicillin and streptomycin were purchased from GIBCO (Grand Island, NY, USA). BrdU and Ki67 Cell Proliferation Kit for high content screening (HCS) was from Cellomics, Inc (Pittsburgh, PA, USA). B16-F10-Luc-G5 was from Caliper Life Sciences (Hopkinton, MA, USA). The vimentin specific antibody was from Beijing Biosynthesis Biotechnology (Beijing, China). Heparin sodium salt from porcine intestinal mucosa was purchased from Sigma-Aldrich, Inc (Saint Louis, MO, USA).

\section{Cell culture}

PC-3M cells were cultured in DMEM medium containing $10 \%$ heat-inactivated FBS, $100 \mathrm{U} / \mathrm{mL}$ penicillin and $100 \mu \mathrm{g} / \mathrm{mL}$ streptomycin in a humidified incubator with $5 \% \mathrm{CO}_{2}$ in air at $37^{\circ} \mathrm{C}$. B16-F10-Luc-G5 cells were cultured in DMEM medium containing $10 \%$ heat-inactivated FBS, $100 \mathrm{U} / \mathrm{mL}$ penicillin and $100 \mu \mathrm{g} / \mathrm{mL}$ streptomycin in a humidified incubator with $5 \% \mathrm{CO}_{2}$ in air at $37^{\circ} \mathrm{C}$.

\section{Cell proliferation}

PC-3M cells were cultured in 96-well plates. When the cells reached approximately $70 \%$ confluency, different doses of heparin $(0,0.5,1,5,25$, and $125 \mu \mathrm{g} / \mathrm{mL})$ were added to the culture and incubated for $24 \mathrm{~h}$. Every group has six wells. Each independent experiment was performed three times. MTS assays were then conducted using the CellTiter96 Aqueous Non-Radioactive Cell Proliferation Assay kit (Promega Corp, Madison, WI, USA), according to the manufacturer's instructions. Absorbance was measured at a wavelength of $490 \mathrm{~nm}$, and the absorbance values of treated cells were calculated as a percentage of the absorbance of untreated cells.

\section{Cell cycle and apoptosis analysis}

For flow cytometric analysis of DNA content, heparin was added to PC-3M cells in the mid-logarithmic phase. After $24 \mathrm{~h}$, cells were collected, pelleted, washed with phosphatebuffered saline (PBS) and resuspended in PBS containing 20 $\mathrm{mg} / \mathrm{L}$ propidium iodide (PI, Sigma, St Louis, MO, USA) and $1 \mathrm{~g} / \mathrm{L}$ ribonuclease A (RNAase A). Fixed cells for each experimental condition were examined by flow cytometry.

For analysis of apoptosis after incubation with heparin for $24 \mathrm{~h}, \mathrm{PC}-3 \mathrm{M}$ cells $\left(1 \times 10^{6}\right.$ cells per treatment condition) were fixed and stained with $5 \mathrm{~mL}$ Annexin V-FITC (BD PharMingen, San Diego, CA) and $5 \mathrm{~mL}$ PI. Flow cytometric analysis was performed on $1 \times 10^{4}$ cells per sample and analyzed using a FACS Calibur (Becton Dickinson, Franklin Lakes, NJ) with a single laser emitting excitation light at $488 \mathrm{~nm}$.

\section{Multiparametric proliferation assay with a HCS analyzer}

The 5-bromo-2'-deoxyuridine (BrdU) and Ki67 Cell Proliferation Kit for HCS reagent kit was used for simultaneous quantification of DNA replication and Ki67 proliferation marker in the same cell. It allows direct measurements of BrdU incorporation and Ki67 expression using a fixed end-point assay based on immunofluorescence detection in cells grown on standard high-density microplates. The primary antibodies were directed toward BrdU and Ki67 (mouse monoclonal and rabbit polyclonal antibodies, respectively) and secondary antibodies were conjugated with DyLight Fluor 488 (green) and DyLight Fluor 549 (orange). Cells were stained and fixed, and total cells per well of the microplates were analyzed using an ArrayScan HCS system (Cellomics Inc., Pittsburgh, PA, USA).

\section{In vivo metastasis}

B16-F10-luc-G5 cells that had been engineered to stably express firefly luciferase (Xenogen Corporation, Alameda, CA, USA) were injected into the lateral tail vein $\left(5 \times 10^{6}\right.$ cells/ $100 \mu \mathrm{L} /$ mouse) of 6-week-old BALB/c nude mice. Mice were anesthetized and given $150 \mathrm{mg} / \mathrm{g}$ of $D$-luciferin in PBS by intraperitoneal injection. Bioluminescence was imaged with a charge-coupled device camera (IVIS; Xenogen) at 10 min after injection. Mice were anesthetized using 1\%-3\% isoflurane (Abbott Laboratories, Chicago, IL, USA). Bioluminescence from relative optical intensity was defined manually, and data were expressed as photon flux normalized to background photon flux, which was defined from relative optical intensity obtained from a control mouse not injected with luciferin. Animal handling and procedures were approved by the Peking University Health Science Center Institutional Animal Care and Use Committee. All animal studies conformed to the principles outlined in the Declaration of Helsinki. 
Activated partial thromboplastin time (APTT) and hemorheological measurements

On day 14, blood specimens of BALB/c nude mice injected with B16-F10-luc-G5 cells were collected by retro-orbital bleeding after anesthesia with ether. Anesthesia was achieved by placing the mouse in a container containing cotton wool soaked in ether. The anesthetic plane was assessed by pinching the toe, tail or ear of the animal. The respiration rate was even and the color of the mucus membranes was bright pink. As the blood was collected, there was no evidence of pain observed in the animals. Whole blood with sodium citrate anticoagulant (9:1), centrifuged at $3000 \mathrm{r} / \mathrm{min}$ for 10 min, and upper plasma was used to detect APTT. APTT was measured in $50 \mu \mathrm{L}$ plasma using C2000-4 Type Coagulometer (Beijing Precil Instrument Co, Ltd). Whole blood with sodium citrate anticoagulant was used for hemorheological measurements. Whole blood viscosity was detected in $800 \mu \mathrm{L}$ of blood by using a viscosity meter (LBY-N6C, Precil Group, Beijing, China). Red blood cell deformability was determined by measuring the elongation index (EI) and orientation index (OI) using an ektacytometer (LBY-BX2 Precil Group, Beijing, China). Blood samples diluted with polyvinyl pyrolidone (PVP) were added to the base plate of the instrument. The EI and OI values were measured under stress rates of 100/s and 1000/s. Hematocrit of red blood cells was measured by aspirating blood into a capillary tube, sealing off the end of the tube and then centrifuging it at $10000 \mathrm{r} / \mathrm{min}$ for $5 \mathrm{~min}$. The height of the red blood cells deposited at the bottom end of the tube was recorded.

Two-dimensional gel electrophoresis (2-DE) and image analysis Monolayers of PC-3M cells were treated with or without hepa$\operatorname{rin}(125 \mu \mathrm{g} / \mathrm{mL})$ for $24 \mathrm{~h}$. Cells were harvested using homemade scrapers. The resulting pellets were resuspended in $100 \mathrm{~mL}$ of solubilization buffer containing $40 \mathrm{mmol} / \mathrm{L}$ Tris, 8 $\mathrm{mol} / \mathrm{L}$ urea, $4 \% w / v$ CHAPS, $60 \mathrm{mmol} / \mathrm{L}$ DTT and $1 \mathrm{mmol} / \mathrm{L}$ PMSF. After three freeze-thaw cycles, samples were centrifuged at $17000 \times \mathrm{g}$ for $15 \mathrm{~min}$, and the supernatants were collected. Another pair of samples tested were the frozen mice lung tissues, which were thawed at room temperature before total proteins were extracted as mentioned above and the protein concentration measured by the Bradford method. First dimension isoelectric focusing (IEF) was performed using linear immobilized $\mathrm{pH}$ gradient readystrips $(24 \mathrm{~cm}, \mathrm{pH} 3-10$, Bio-Rad Laboratories, Inc, Hercules, CA, USA). Protein samples $(500 \mu \mathrm{g})$ from each group were solubilized in rehydration buffer [ $8 \mathrm{~mol} / \mathrm{L}$ urea, $4 \%$ CHAPS, $65 \mathrm{mmol} / \mathrm{L}$ DTT, 0.2\% BioLyte (Bio-Rad) and $0.001 \%$ bromothymol blue] to a volume of $125 \mathrm{~mL}$ and allowed to incubate at room temperature for $30 \mathrm{~min}$. After positive rehydration for $12 \mathrm{~h}$ at $50 \mathrm{~V}$, IEF was run at $20^{\circ} \mathrm{C}$ in the following steps: $250 \mathrm{~V}$ linear for $30 \mathrm{~min}, 500$ $\mathrm{V}$ rapid for $30 \mathrm{~min}, 4000 \mathrm{~V}$ linear for $3 \mathrm{~h}, 4000 \mathrm{~V}$ rapid until $20000 \mathrm{~V}$. The IEF strips were then equilibrated by serial incubation (15 min) in equilibration buffer $(6 \mathrm{~mol} / \mathrm{L}$ urea, $2 \% \mathrm{SDS}$, $0.375 \mathrm{~mol} / \mathrm{L}$ Tris- $\mathrm{HCl}$ at $\mathrm{pH} 8.8,20 \%$ glycerol and $20 \mathrm{mg} / \mathrm{mL}$ DTT) and in equilibration buffer containing $2.5 \%$ iodoacet- amide instead of DTT. Subsequently, the samples were separated in the second dimension on $12 \%$ polyacrylamide gels at $80 \mathrm{~V}$ for $10 \mathrm{~min}$ and then at $110 \mathrm{~V}$ for $50-60 \mathrm{~min}$. Gels were stained with Coomassie Blue. The differentially expressed protein spots were excised manually from the sodium dodecyl sulphate-polyacrylamide gel (SDS-PAGE) and subjected to ingel tryptic digestion.

\section{Mass spectrometry identification of proteins}

Proteins of interest were analyzed by nanoelectrospray with a hybrid quadrupole time-of-flight (Q-TOF) mass spectrometer (Waters, Milford, MA, USA). The peptide mixture was carried out on a Waters Capillary liquid chromatography system including three pumps, A, B, and C (Waters). Fused silica tubing were packed with Symmetry $300^{\mathrm{TM}} \mathrm{C} 18,3.5 \mathrm{~mm}$ spherical particles with a pore diameter of 1008 (Waters). The flow rate was set at $2.5 \mathrm{~mL} / \mathrm{min}$. Samples were injected at a flow rate of $20 \mathrm{~mL} / \mathrm{min}$.

\section{Western blot analysis}

Equal amounts of protein were analyzed by SDS-PAGE on a $10 \%$ polyacrylamide gel for vimentin and 14-3-3 zeta/delta and transferred to a polyvinylidene difluoride membrane (Millipore Corp, Bedford, MA, USA). The membrane with blotted proteins was blocked for $1 \mathrm{~h}$ with blocking buffer containing 5\% non-fat dry milk and $0.05 \%$ Tween 20 in Trisbuffered saline (TBS-T), followed by incubation with vimentin antibody diluted (1:100) in blocking buffer overnight at $4{ }^{\circ} \mathrm{C}$. The membrane was then washed three times with TBS-T for 30 min and incubated at room temperature for $1 \mathrm{~h}$ with diluted (1:2000) secondary AP-labeled IgG (Santa Cruz Biotechnology, Santa Cruz, CA, USA). Blots were extensively washed in TBST buffer and detection was done using the NBT/BCIP reaction (Amersco, NJ, USA) ${ }^{[7]}$.

\section{Ribonucleic acid isolation and reverse transcription-polymerase} chain reaction (RT-PCR)

Total RNA was isolated using Trizol reagent (Invitrogen, Groningen, the Netherlands). First-strand cDNAs were generated from RNA samples by reverse transcription using oligo (dT). The following primers were used to amplify fragments of $\beta$-actin: 5'-ATCATGTTTGAGACCTTCAACA-3' (sense) and 5'-CATCTCTTGCTCGAAGTCCA-3' (antisense); transforming growth factor (TGF)- $\beta$ : $5^{\prime}$-CAAGTGGACATCAAC GGTGAGG-3' (sense) and 5'-TGGCCATGAGAAGCAGGAAAGG-3' (antisense); vimentin: 5'-GACAATGCGTCTCTGGCACGTCTT-3' (sense) and 5'TCCTCCGCCTCCTGCAGGTTCTT-3' (antisense); $a_{v}$-integrin: 5'-GACTGTGTGGAAGACAATGTCTGTAAACCC-3' (sense) and 5'-CCAGCTAAGAGTTGAGTTCCAGCC-3' (antisense); E-cadherin: 5'-TGGAGGAATTCTTGCTTTGC-3' (sense) and 5'-CGTACATGTCAGCCAGCTTC-3' (antisense).

\section{Short interfering ribonucleic acid (siRNA) transfection}

siRNA duplexes used in this study were bought from Santa Cruz, to interfere with vimentin expression. Non-silencing 
siRNA (5'-UUAAGUAGCUUGGCCUUGATdT-3' and 5'-UCAAGGCCAAGCUACUUAATdT-3') was used as the negative controls. siRNA duplexes were transfected into HUVECs with siRNA transfection reagent (Polyplus-transfection Inc, San Marcos, CA, USA), according to the manufacturer's instructions. After transfection for $48 \mathrm{~h}$, cells were subjected to multiparametric proliferation assay with a HCS analyzer.

\section{Statistical analysis}

Data were expressed as means \pm standard error of mean (SEM). Statistical significance of differences between means was determined by one-way analysis of variance (ANOVA) followed by Dunnett's test (Graphpad Prism 5 software). $P<0.05$ was considered to be statistically significant.

\section{Results}

\section{Effect of heparin on PC-3M cell viability and cell cycle}

As estimated by the MTS assay, cell viability decreased dose dependently after $24 \mathrm{~h}$ of exposure to heparin at different concentrations $(0.5,1,5,25$, and $125 \mu \mathrm{g} / \mathrm{mL}$ ) (Figure 1A). Heparin 5, 25, and $125 \mu \mathrm{g} / \mathrm{mL}$ arrested cells in the $\mathrm{G}_{1}$ phase and influence DNA synthesis, with a significant effect at 125 $\mu \mathrm{g} / \mathrm{mL}(P<0.05$, Figure 1B). Correspondingly, cells in the $S$ phase decreased with increasing doses of heparin.

\section{Effect of heparin on apoptosis of PC-3M cells}

Annexin $\mathrm{V}^{+}$and $\mathrm{PI}^{-}$staining represented early apoptotic cells, and Annexin $\mathrm{V}^{+}$and $\mathrm{PI}^{+}$staining represented late apoptotic and necrotic cells. Although the apoptotic cells were slightly elevated in the $125 \mu \mathrm{g} / \mathrm{mL}$ heparin treated group compared with the vehicle treated group, the difference was not significant (control: $1.55 \% \pm 0.19 \%$ vs $5 \mu \mathrm{g} / \mathrm{mL}$ heparin: $1.44 \% \pm 0.06 \%$, $25 \mu \mathrm{g} / \mathrm{mL}$ heparin: $2.21 \% \pm 0.08 \%, 125 \mu \mathrm{g} / \mathrm{mL}$ heparin: $5.91 \% \pm 0.15 \%$ ) (Figure 1C).

\section{Effect of heparin on BrdU incorporation and Ki67 expression in} PC-3M cells

BrdU incorporation and Ki67 activation in PC-3M cells were measured simultaneously in the same cells as described in Methods. As an alternative to $\left[{ }^{3} \mathrm{H}\right]$ thymidine, BrdU, a thymidine analog, enables detection of DNA replication in actively proliferating cells using a monoclonal antibody directed against BrdU and fluorophore-conjugated secondary antibody. In addition, Ki67 antigen-positive cells can provide a specific and accurate indication of proliferating cells. As determined by HCS, we found that heparin suppressed BrdU incorporation and Ki67 expression in PC-3M cells compared to the control group, with statistically significant differences seen at the dosage of $125 \mu \mathrm{g} / \mathrm{mL}$ (Figure 1D).

\section{Heparin inhibits B16-F10-luc-G5 cell lung metastasis in Balb/c nude mice}

Balb/c nude mice were implanted with B16-F10-luc-G5 carcinoma cells by tail vein injection and systemically treated with vehicle (model), $30 \mathrm{mg} / \mathrm{kg}$ heparin intravenously 30 min before B16-F10-luc-G5 carcinoma cell injection and $24 \mathrm{~h}$ after B16-F10-luc-G5 carcinoma cell injection. From day 2, the growth of B16-F10-luc-G5 tumor burden over time in representative Balb/c nude mice was measured by in vivo imaging. By this analysis, progression of tumors in the whole body of mice and lung tissue was delayed and reduced in the $30 \mathrm{mg} / \mathrm{kg}$ heparin treatment group, and the growth of the primary tumor was reduced significantly from day 8 onward when detected from the ventral $(\mathrm{A}, \mathrm{B}, \mathrm{C})$ and dorsal $(\mathrm{D}, \mathrm{E}, \mathrm{F})$ views $(P<0.01$, Figure 2$)$.

Heparin influences APTT and hemorheological parameters of Balb/c nude mice burdened with B16-F10-luc-G5 cells

At the 100/s and 1000/s shear rate, heparin could obviously decrease the whole blood viscosity, and there were significant differences when the tumor burdened (model) group was compared with the normal group or the heparin treated group $(P<0.01$, Table 1$)$. Factors influencing whole blood viscosity were measured including red blood hematocrit, EI and OI. We also found that in the model group, red blood hematocrit, EI and OI were decreased significantly compared with the normal group $(P<0.01)$ at the $100 / \mathrm{s}$ shear rate. At the shear rate of $1000 / \mathrm{s}$ in the model group, OI was decreased significantly compared with the normal group $(P<0.01)$, but no significant difference was detected for EI. Additionally, heparin maintained relatively normal red blood hematocrit, with EI and OI at the $100 / \mathrm{s}$ shear rate and OI at the 1000 /s shear rate being significantly different with the model group $(P<0.01$, Table 1). We had not found the difference in APTT between normal Balb/c nude mice with those burdened with B16-F10-luc-G5 cells; and heparin had also no influence on APTT of Balb/c nude mice burdened with B16-F10-luc-G5 cells.

\section{Comparative proteomic analysis of PC-3M cells with or without heparin treatment}

To explore the molecular mechanisms by which heparin exerts

Table 1. Effect of heparin on activated partial thromboplastin time (APTT) and hemorheological parameters in Balb/c nude mice burdened with B16F10-Iuc-G5 cells. Mean \pm SEM. $n=5$. ${ }^{\circ} P<0.01$ vs model.

\begin{tabular}{|c|c|c|c|c|c|c|c|c|}
\hline Drug & Hematocrit (\%) & $100 / \mathrm{s}$ & $1000 / \mathrm{s}$ & $100 / \mathrm{s}$ & $1000 / s$ & $100 / \mathrm{s}$ & $1000 / \mathrm{s}$ & APTT (s) \\
\hline Normal & $39 \pm 3.99^{c}$ & $36.61 \pm 5.58^{c}$ & $66.9 \pm 4.16$ & $8.2 \pm 0.74^{c}$ & $10.1 \pm 1.15^{c}$ & $7.77 \pm 0.64^{c}$ & $3.54 \pm 0.47^{c}$ & $28.6 \pm 4.35$ \\
\hline Model & $21 \pm 4.60$ & $28.2 \pm 2.69$ & $64.2 \pm 3.58$ & $4.94 \pm 0.69$ & $5.03 \pm 0.24$ & $12.19 \pm 2.28$ & $4.58 \pm 0.02$ & $27.11 \pm 3.13$ \\
\hline Heparin & $44 \pm 4.55^{c}$ & $37.6 \pm 4.60^{\circ}$ & $65.6 \pm 4.80$ & $8.54 \pm 0.83^{c}$ & $9.34 \pm 0.91^{\circ}$ & $8.06 \pm 0.13^{c}$ & $3.88 \pm 0.11^{c}$ & $29.02 \pm 4.08$ \\
\hline
\end{tabular}


A

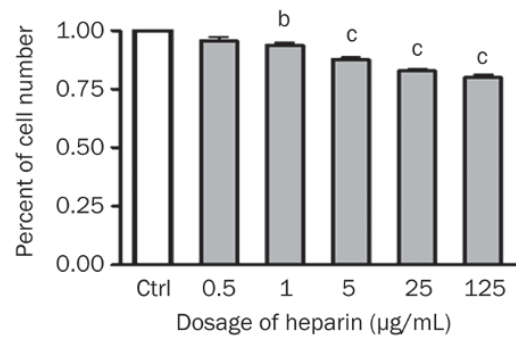

B
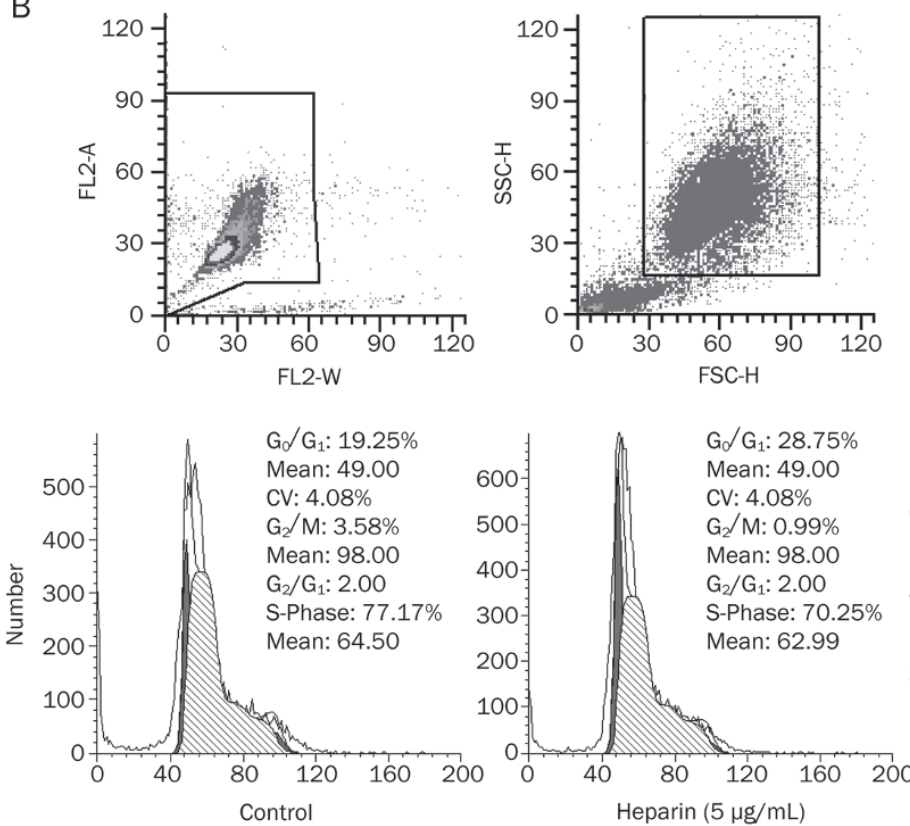
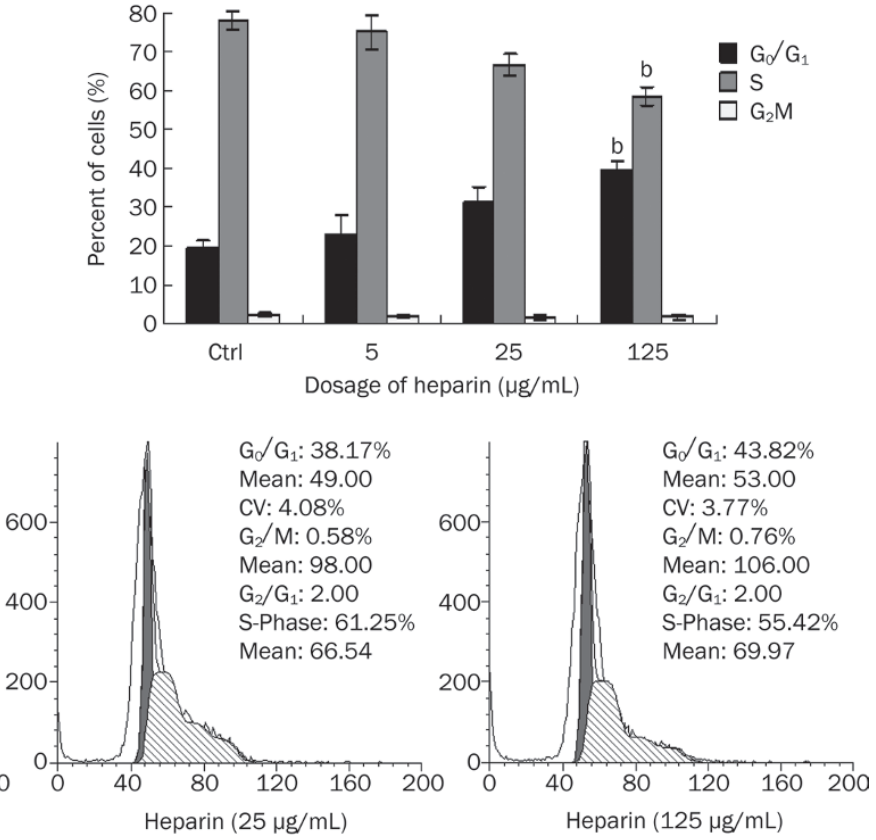

Channels (FL2-A)

C
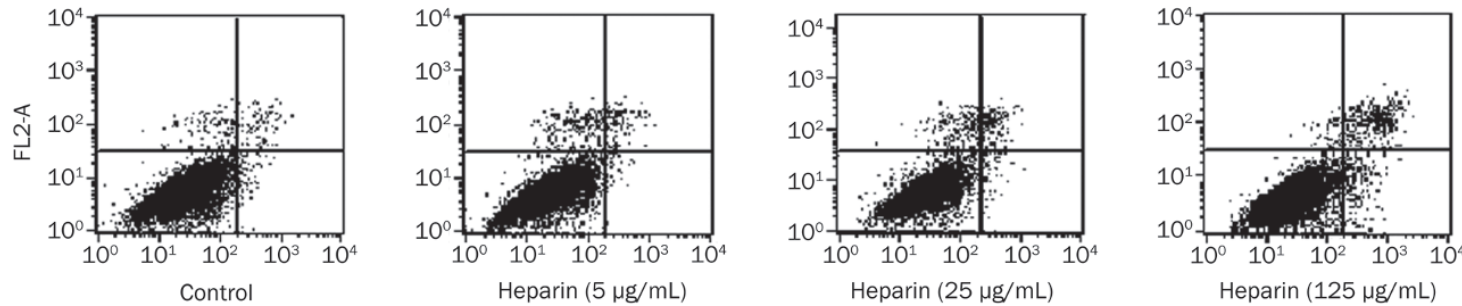

FL1-H
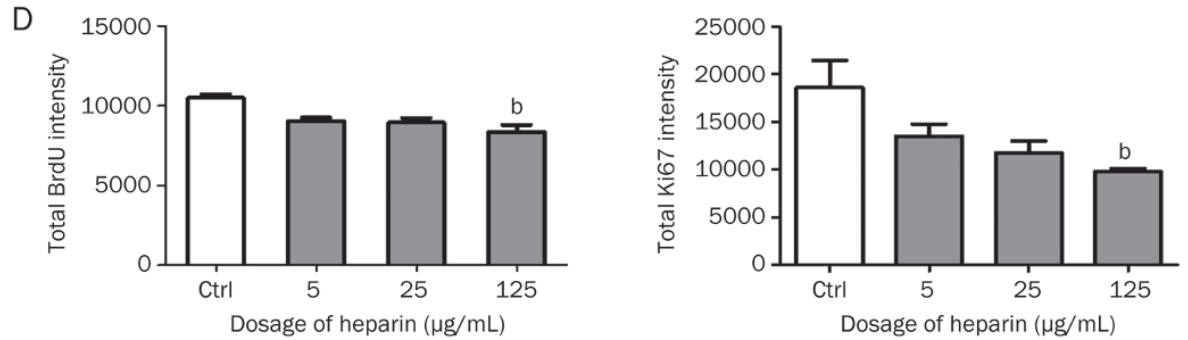

Figure 1. Heparin inhibits PC-3M cell growth. (A) MTS assays demonstrated a heparin-induced, dose-dependent decrease in PC-3M cell growth. (B) Flow cytometry showed that heparin treatment $(5,25$, and $125 \mu \mathrm{g} / \mathrm{mL}, 24 \mathrm{~h})$ resulted in marked cell cycle changes. (C) Flow cytometric analysis by Annexin V and PI double staining showed that heparin treatment (5, 25, and $125 \mu \mathrm{g} / \mathrm{mL}, 24 \mathrm{~h}$ ) induced mild cell apoptosis. (D) HCS analysis showed that heparin suppressed BrdU incorporation and Ki67 expression in PC-3M cells. A total of 3000 cells were plated per well and incubated for $24 \mathrm{~h}$ before treatment with 5,25 , and $125 \mu \mathrm{g} / \mathrm{mL}$ heparin for $24 \mathrm{~h}$. Control wells were treated with PBS per well. BrdU incorporation and Ki67 expression were measured simultaneously. Mean \pm SEM. $n=6$. ${ }^{b} P<0.05,{ }^{\circ} P<0.01$ compared with control. Each independent experiment was performed three times. 

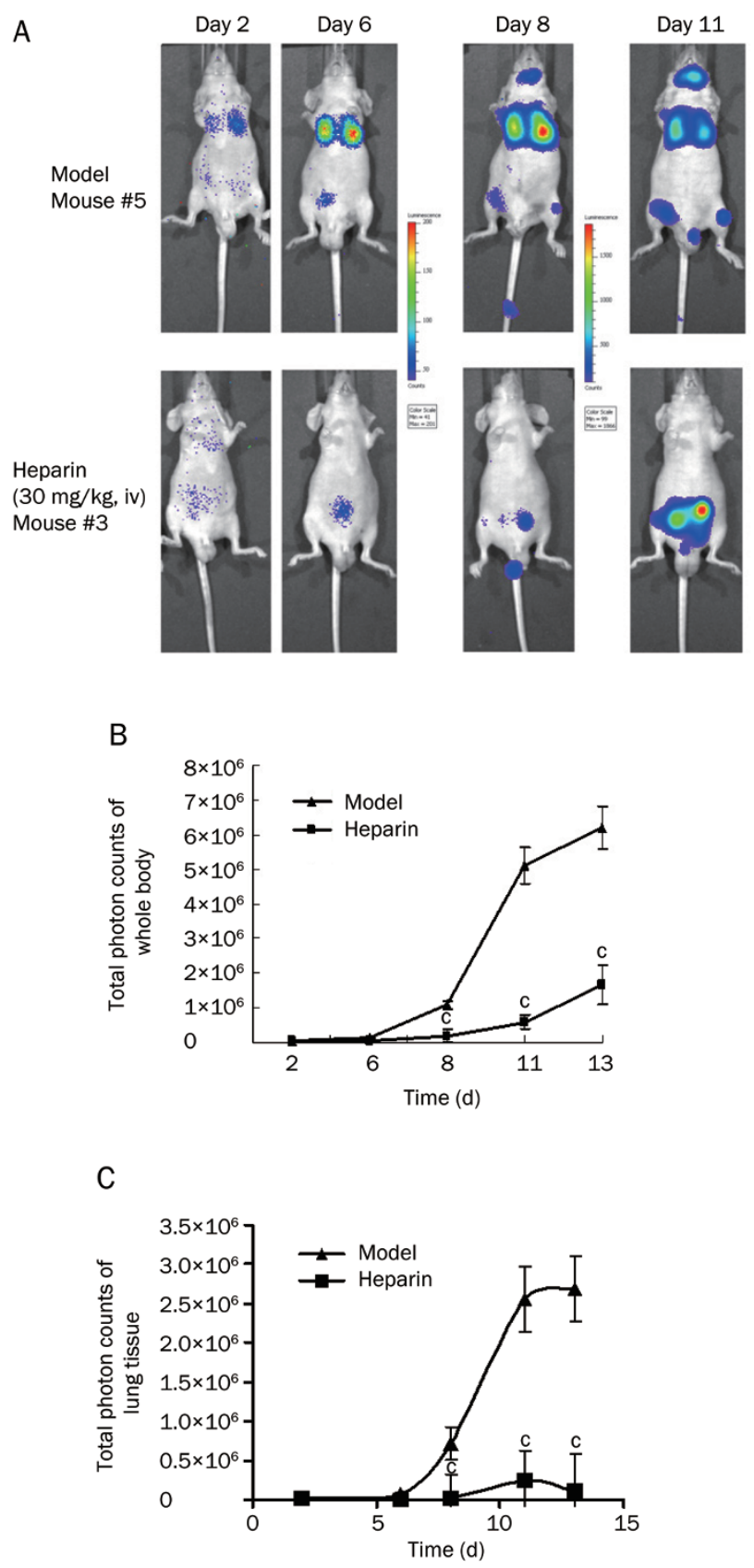
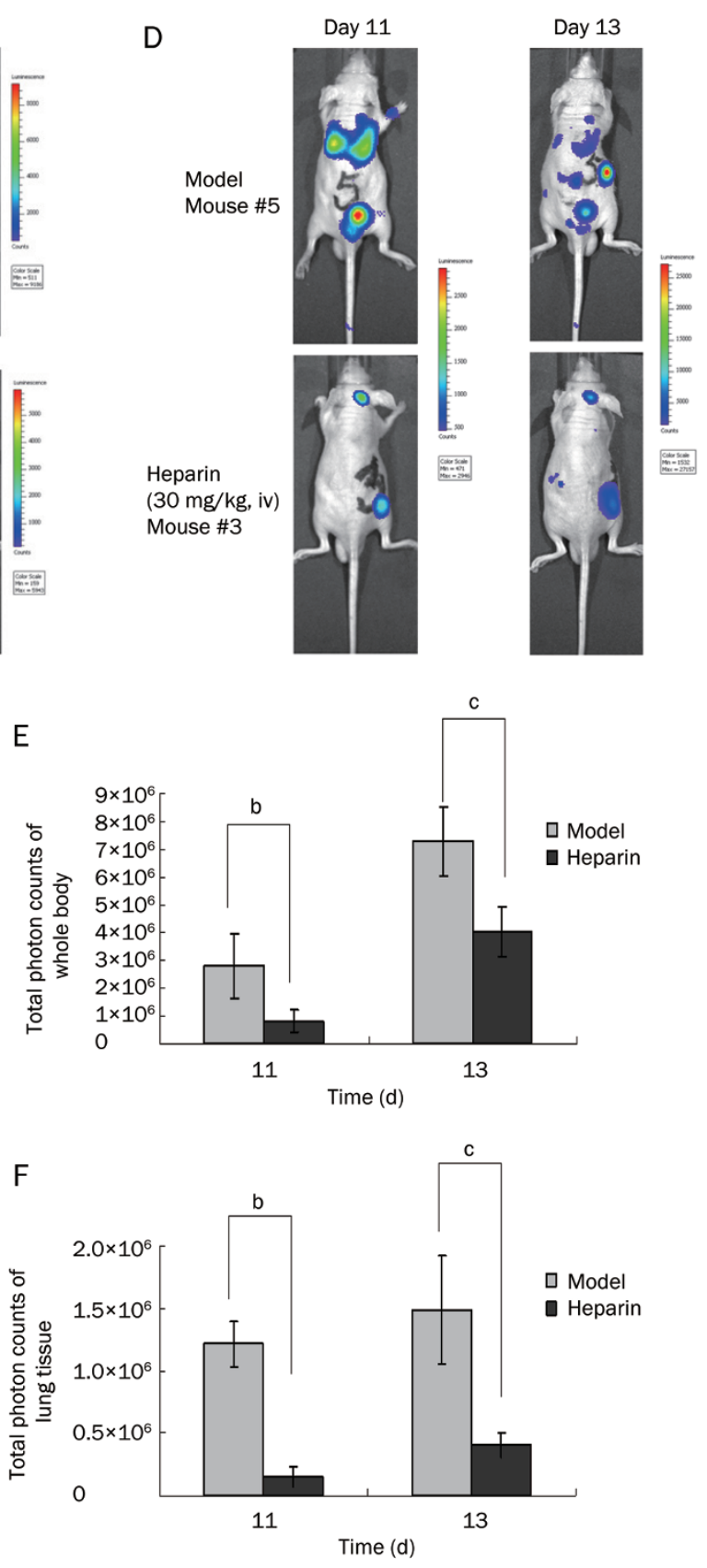

Figure 2. In vivo imaging of B16-F10-luc-G5 cell-derived tumors in mice. The cells $\left(5 \times 10^{6}\right)$ were injected intravenously through the tail vein of male $B A L B / c$ nude mice. Mice were imaged in vivo from the ventral (A) and dorsal (D) views between 2 to 14 days after the injection. Shown are images of one representative control (saline) animal and one representative heparin-treated mouse. Images were set on a pseudocolor scale to show relative bioluminescent changes over time. Metastatic signals reappeared on the day following administration of control mice with vehicle and multiple signals developed on day 6. In vivo imaging confirmed metastases in lungs (highest signal), ileum, jaws and skin (lower signal). Mice administered heparin (30 mg/kg, $30 \mathrm{~min}$ before and $24 \mathrm{~h}$ after tumor cell injection via the tail vein) showed significant reduction of pulmonary metastatic nodules. B, C, E, F show the statistical analysis of the total photon counts of whole body (B, E) or lung tissue (C, F) of BALB/C nude mice burdened with B16-F10-luc-G5 cells from the ventral (B, C) and dorsal (E, F) views. Mean \pm SEM. $n=3 .{ }^{b} P<0.05,{ }^{C} P<0.01$ compared with the vehicle-treated control group.

anti-tumor and anti-proliferative effects in PC-3M cells, we compared the protein expression patterns in heparin treated $(125 \mu \mathrm{g} / \mathrm{mL})$ and untreated cells. 2-DE analysis showed generally similar protein expression patterns in the heparin treated and untreated PC-3M cells (Figure 3A, B). The protein spots were analyzed by PDQuest software, and 83 protein spots were significantly decreased in heparin treated versus untreated PC-3M cells ( $P<0.05$, Supporting information). Thirty of these obviously decreased spots after heparin treatment were identified by Q-TOF analysis (Table 2). 
Table 2. Thirty of the significantly decreased spots after heparin treatment in PC-3M cells identified by Q-TOF analysis and search of the Mascot database.

\begin{tabular}{|c|c|c|c|c|c|c|}
\hline $\begin{array}{l}\text { Protein } \\
\text { spot }\end{array}$ & Protein name & Mass & Score & Matches & Sequences & emPAl \\
\hline 1 & Anterior gradient protein 2 (AGR2) & 20024 & 497 & $27(17)$ & $8(6)$ & 3.06 \\
\hline 2 & Adenylate kinase isoenzyme 1 (KAD1) & 21735 & 190 & $9(5)$ & $6(5)$ & 1 \\
\hline 3 & GTP-binding nuclear protein Ran (RAN) & 24579 & 694 & $29(24)$ & $12(10)$ & 3.63 \\
\hline 4 & 14-3-3 protein zeta/delta 1433Z_HUMAN & 27899 & 89 & $1(1)$ & $1(1)$ & 0.12 \\
\hline 7 & ATP synthase subunit alpha, mitochondrial (ATPA_HUMAN) & 59828 & 240 & $24(13)$ & $13(8)$ & 0.53 \\
\hline 8 & ATP synthase subunit alpha, mitochondrial ATPA_HUMAN & 59828 & 29 & $2(1)$ & $2(1)$ & 0.98 \\
\hline 9 & Annexin A11ANX11_HUMAN & 54697 & 724 & $19(16)$ & $8(7)$ & 0.57 \\
\hline 10 & Vimentin & 55123 & 1124 & $51(29)$ & $21(14)$ & 1.84 \\
\hline 11 & Dihydrolipoyl dehydrogenase, mitochondrial DLDH_HUMAN & 54713 & 283 & $12(10)$ & $6(4)$ & 1.23 \\
\hline 15 & Stress-70 protein, mitochondrial GRP75_HUMAN & 73920 & 149 & $3(3)$ & $2(2)$ & 0.09 \\
\hline 16 & L-lactate dehydrogenase B chain LDHB_HUMAN & 36900 & 193 & $6(5)$ & $5(4)$ & 0.46 \\
\hline 17 & T-complex protein 1 subunit zeta TCPZ_HUMAN & 58444 & 343 & $12(9)$ & $5(4)$ & 0.39 \\
\hline 18 & Heat shock protein beta-1 HSPB1_HUMAN & 22826 & 1172 & $36(27)$ & $12(10)$ & 4.91 \\
\hline 19 & T-complex protein 1 subunit gamma TCPG_HUMAN & 61066 & 103 & $4(3)$ & $3(2)$ & 1.55 \\
\hline 20 & Pyruvate kinase isozymes M1/M2 KPYM_HUMAN & 58470 & 88 & $3(2)$ & $3(2)$ & 1.21 \\
\hline 21 & RuvB-like 2 RUVB2_HUMAN & 51296 & 262 & $9(7)$ & $6(5)$ & 0.45 \\
\hline 22 & Phosphoglycerate mutase 1 PGAM1_HUMAN & 28900 & 420 & $17(11)$ & $7(7)$ & 1.67 \\
\hline 23 & Elongation factor Tu, mitochondrial EFTU_HUMAN & 49852 & 1519 & $42(31)$ & $15(11)$ & 1.79 \\
\hline 24 & Heat shock protein HSP 90-alpha HS90A_HUMAN & 85006 & 1345 & $41(31)$ & $26(20)$ & 1.48 \\
\hline 25 & Heat shock protein $75 \mathrm{kDa}$, mitochondrial TRAP1_HUMAN & 80345 & 285 & $4(4)$ & $1(1)$ & 0.04 \\
\hline
\end{tabular}
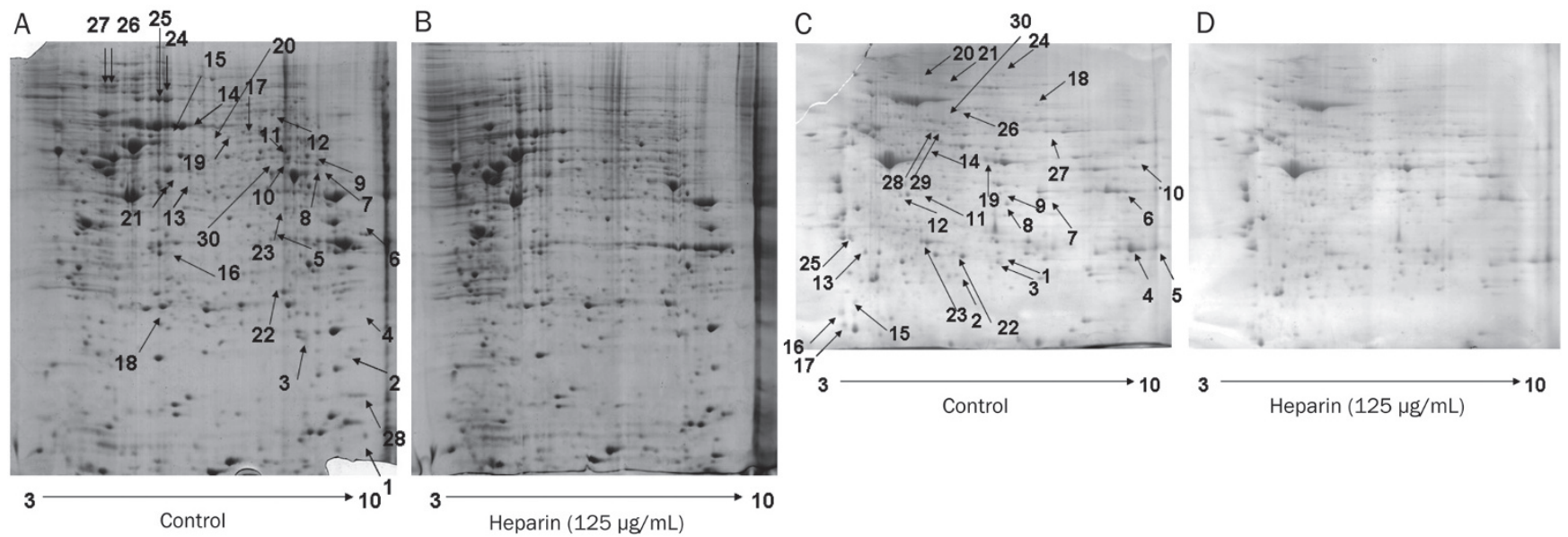

Figure 3. 2-DE analysis of protein expression in PC-3M cells and lungs of mice burdened with B16-F10-luc-G5 cells with or without heparin treatment. Representative 2-DE maps of proteins in PC-3M cell lysis without (A) or with heparin treatment (B). Representative 2-DE maps of proteins in lysed lung tissues of mice bearing B16-F10-luc-G5 cells without (C) or with (D) heparin administration. Approximately $500 \mu \mathrm{g}$ of total protein were focused on linear immobilized $\mathrm{pH}$ gradient strips $(\mathrm{pH} 3-10,24 \mathrm{~cm}$ ) before separating on a $12 \%$ sodium dodecyl sulphate-polyacrylamide gel and staining with Coomassie blue for detecting protein spots. The experiment was performed three times. 
Comparative proteomic analysis of lungs of mice bearing B16F10-luc-G5 cells with or without heparin administration

To further elucidate the possible novel molecular mechanisms by which heparin might exert its anti-tumor and anti-metastasic effects in Balb/c nude mice burdened with B16-F10luc-G5 cells, we compared protein expression patterns in the lungs of heparin-treated and untreated (control) mice. 2-DE analysis showed generally similar protein expression patterns in the heparin-treated and untreated groups (Figure $3 \mathrm{C}$ and 3D). Among them, 30 obviously different spots (indicated by arrows) were identified by using Q-TOF analysis (Table 3).

Effect of heparin on expression levels of 14-3-3 zeta/delta, vimentin, E-cadherin, TGF- $\beta$, and $\alpha_{v}$-integrin in PC-3M cells

After comparing the 2-DE results from PC-3M cells and lungs of mice burdened with B16-F10-luc-G5 cells, we found 14-3-3 zeta/delta and vimentin protein spots were identified in common. Then, our Western blot analysis confirmed that heparin could dose-dependently decrease the protein expression of 14-3-3 zeta/delta and vimentin, and there were significant differences at 25 and $125 \mu \mathrm{g} / \mathrm{mL}$ (Figure 4A). Cytoskeleton protein vimentin is involved in tumor mobility, adhesion, and epithelial to mesenchymal transition (EMT). Vimentin also co-localizes with $a_{v} \beta_{3}$-integrin (focal contacts) when tumor cell moved and thought as an important marker of tumor cell EMT, along with E-cadherin, TGF- $\beta$. To determine whether E-cadherin, TGF- $\beta$ and $\alpha_{v}$-integrin are transcriptionally regulated by heparin, RT-PCR analysis was performed on heparin treated PC-3M cells. Vimentin and E-cadherin mRNA decreased at the high dosage of heparin, while TGF- $\beta$ mRNA increased with heparin treatment in the PC-3M cells (Figure $4 \mathrm{~B})$, although the differences were not significant. Expression of $a_{v}$-integrin mRNA in PC-3M cells decreased with heparin 25 and $125 \mu \mathrm{g} / \mathrm{mL}$ treatment (Figure $4 \mathrm{C}$ ).

The effect of vimentin-targetted siRNA on PC-3M cell proliferation When PC-3M cells were transfected with siRNA against the vimentin gene for $48 \mathrm{~h}$, the expression of vimentin in cells was partially reduced by $35 \%$ in comparison to the controls $(P<0.05$; Figure 5A) and the BrdU incorporation and Ki67 activation in PC-3M cells was reduced significantly compared with the control $(P<0.05$; Figure 5B).

Table 3. Thirty of the significantly decreased spots after heparin administration in lung tissue of Balb/c nude mice burdened with B16-F10-luc-G5 cells identified by Q-TOF analysis and search of the Mascot database.

\begin{tabular}{|c|c|c|c|c|c|c|}
\hline $\begin{array}{l}\text { Protein } \\
\text { spot }\end{array}$ & Protein name & Mass & Score & Matches & Sequences & emPAl \\
\hline 1 & Carbonyl reductase [NADPH] & 26056 & 335 & $15(9)$ & $7(5)$ & 1.32 \\
\hline 2 & 14-3-3 protein zeta/delta & 27925 & 370 & $15(11)$ & $10(7)$ & 2.08 \\
\hline 3 & Hemoglobin subunit beta-1 & 15944 & 79 & $1(1)$ & $1(1)$ & 0.21 \\
\hline 4 & Selenium-binding protein 1 & 53051 & 72 & $4(3)$ & $3(2)$ & 0.13 \\
\hline 7 & Carbonyl reductase [NADPH] 2 & 26056 & 662 & $34(23)$ & $12(7)$ & 1.96 \\
\hline 8 & Troponin I, fast skeletal muscle & 21515 & 268 & $26(15)$ & $9(5)$ & 2.68 \\
\hline 9 & Hemoglobin subunit alpha & 15133 & 165 & $6(6)$ & $3(3)$ & 0.84 \\
\hline 10 & Carbonic anhydrase 3 & 29633 & 144 & $31(11)$ & $8(5)$ & 1.34 \\
\hline 11 & Triosephosphate isomerase & 32684 & 65 & $25(7)$ & $10(4)$ & 0.47 \\
\hline 16 & Monoglyceride lipase & 33708 & 64 & $13(3)$ & $7(2)$ & 0.46 \\
\hline 17 & Fructose-bisphosphate aldolase A & 39787 & 45 & $3(1)$ & $3(1)$ & 0.17 \\
\hline 18 & Creatine kinase M-type & 43246 & 36 & $25(2)$ & $10(1)$ & 0.16 \\
\hline 19 & Advanced glycosylation end product-specific receptor & 42984 & 19 & $2(0)$ & $1(0)$ & 0.08 \\
\hline 20 & Beta-enolase & 47337 & 31 & $9(1)$ & $8(1)$ & 0.07 \\
\hline 21 & Fatty acid-binding protein, epidermal & 15470 & 19 & $1(1)$ & $1(1)$ & 0.22 \\
\hline 22 & $40 \mathrm{~S}$ ribosomal protein SA & 32931 & 28 & $3(1)$ & $3(1)$ & 0.1 \\
\hline 23 & Myosin light chain 3 & 22521 & 635 & $41(30)$ & $14(11)$ & 3.6 \\
\hline 24 & Serum albumin & 70700 & 302 & $25(17)$ & $12(7)$ & 0.44 \\
\hline 25 & Vimentin & 53712 & 24 & $1(0)$ & $1(0)$ & 0.06 \\
\hline 26 & Phosphoglycerate mutase 1 & 28928 & 217 & $19(11)$ & $7(4)$ & 0.92 \\
\hline
\end{tabular}


A
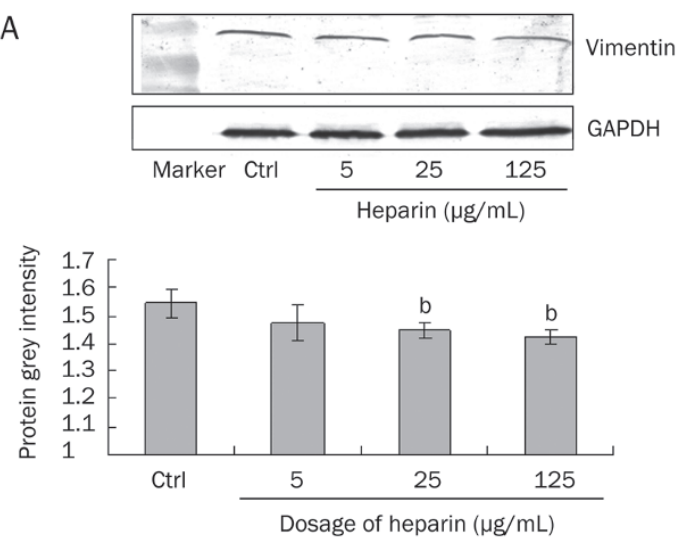

B
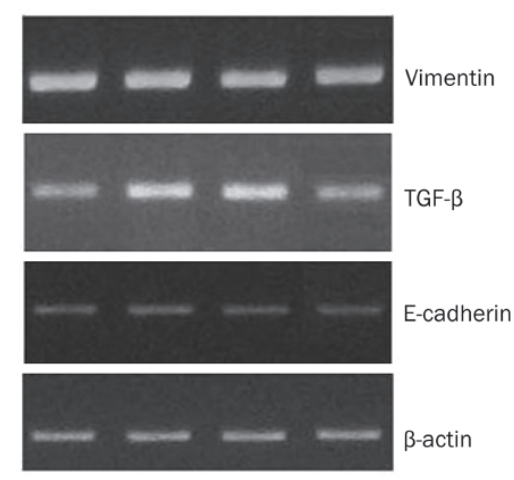

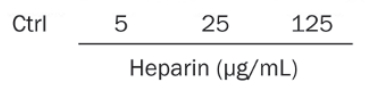

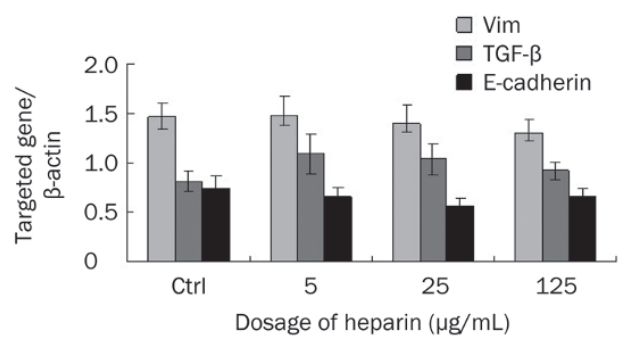

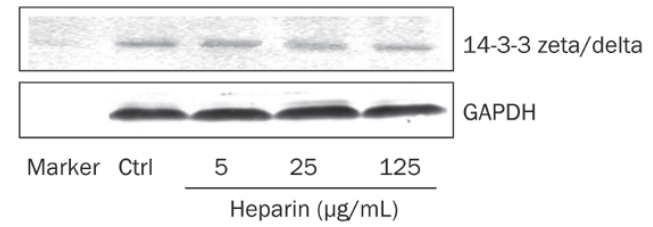

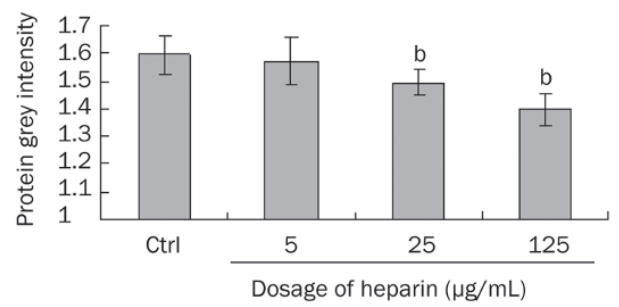

C

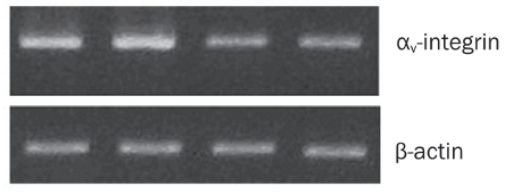

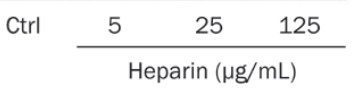

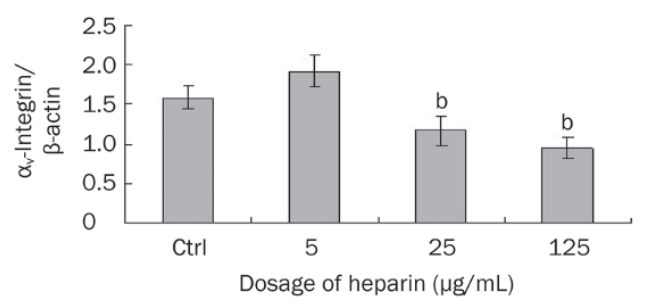

Figure 4. Effects of heparin on protein expression of vimentin and 14-3-3 zeta/delta (A) and mRNA expression of vimentin, TGF- $\beta$, E-cadherin, and $\alpha_{v}-$ integrin (B, C) in PC-3M cells. Mean \pm SEM. $n=3 .{ }^{b} P<0.05$ vs the vehicle-treated control group. Each independent experiment was performed three times.

\section{Discussion}

In the experimental metastasis model, B16F10-luc-G5 tumor cells stably expressing firefly luciferase injected intravenously in the tail vein develop into pulmonary metastasis and are easily detected in vivo over time. We obtained results similar to that of other ${ }^{[8-10]}$ in that a single administration of heparin 30 min before injection of tumor cells into the tail vein could significantly inhibit this lung metastasis. In our study, with a second administration of heparin at $24 \mathrm{~h}$ after tumor cell injection, the lung metastasis could be significantly inhibited throughout the 14-day experimental period. APTT data showed that this injection of heparin was safe. As blood disorders (ie, low hematocrit) have been reported in cancer patients, we also analyzed hemorheological paremeters in Balb/c nude mice burdened with B16-F10-luc-G5 cells. Erythrocyte deformability and hematocrit were decreased, while the whole blood viscosity was increased in the model group compared to those of normal subjects ${ }^{[11,12]}$. The observation that heparin could maintain the normal levels of these blood parameters and also inhibit metastasis as mentioned above indicated that the influence of heparin on circulating cells was related to its inhibition of tumor development.

The effect of heparin on tumors includes interference with cancer-induced hypercoagulation, cancer cell proliferation, degradation of the extra-cellular matrix (involving the heparan sulfate chains of cell surface and extracellular matrix proteoglycans), angiogenesis, selectin-mediated binding of platelets and cancer cells, growth factors and their receptors, hepara- 
A

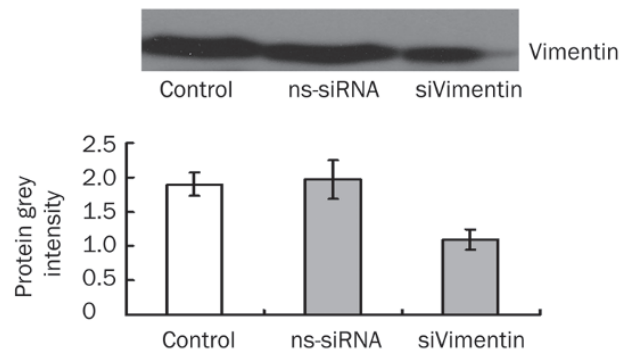

B
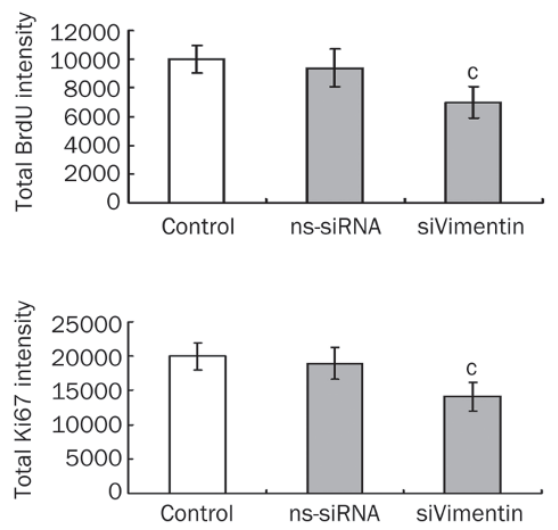

Figure 5. siRNA interferes with vimentin expression in PC-3M cells. Nonsilencing siRNA (ns-siRNA) was used as a negative control. Vimentintargeted siRNA (siVimentin) was transfected into PC-3M for $48 \mathrm{~h}$; the total protein was extracted with protein lysis buffer and protein concentrations were measured using the Bradford protein assay. Cell lysates were analyzed by Western blotting (A). The effect of this vimentin knockdown on BrdU incorporation and Ki67 expression (B) in PC-3M cells. A total of 3000 cells were plated per well and incubated for $24 \mathrm{~h}$. Mean \pm SEM. $n=6$. ${ }^{\mathrm{c}} P<0.01$ compared with control. Each independent experiment was performed three times.

nase, tumor progression, and metastasis ${ }^{[13,14]}$. Heparin was proposed to have a role in maintaining the effects of chemotherapeutic drugs, as both UFH and LMWH have been shown to act as chemotherapy sensitizers ${ }^{[4,5]}$. In our evaluation of the influence of heparin on tumor cell proliferation, cell cycle and apoptosis, we found that it could inhibit proliferation of PC-3M tumor cells and arrest them in the $G_{0} / G_{1}$ phase. Heparin has been reported to induce apoptosis in tumor cells mediated by caspase- 3 , caspase- 8 , Fas, and Bcl- $2^{[15]}$. In this study, we did not find a significant influence of heparin on tumor cell apoptosis, although there was a slight increase in number of apoptotic PC-3M cells treated with heparin $(125 \mu \mathrm{g} / \mathrm{mL})$.

To gain a global overview of protein changes in response to heparin treatment, we utilized the 2-DE method to evaluate PC-3M cells and lungs of mice bearing B16-F10-luc-G5 cells. Our 2-DE analysis identified 60 different down-regulated proteins in PC-3M tumor cells and lungs after heparin treatment. The functions of those differentially expressed proteins are related to cell structure and movement (eg, fascin, transgelin, filamin, myosin, vimentin, dynein), cell growth and apoptosis (eg, anterior gradient protein 2 , pyruvate kinase isozymes
M1/M2, enolase, 14-3-3 zeta/delta), energy metabolism (eg, adenylate kinase isoenzyme 1, ATP synthase subunit alpha, dihydrolipoyllysine-residue succinyltransferase component of 2-ox oglutarate dehydrogenase complex), nuclear activity regulation (eg, GTP-binding nuclear protein Ran) and several chaperones in the control of cell proliferation $(e g$, stress-70 protein, T-complex protein 1, heat shock protein HSP 90, heat shock protein 75 and endoplasmin).

After comparing the 2-DE results from PC-3M cells and lungs of mice burdened with B16-F10-luc-G5 cells, we found five protein spots which were identified in common: adenylate kinase isoenzyme 1, 14-3-3 protein zeta/delta, vimentin, phosphoglycerate mutase 1 and $\beta$-enolase.

Previously, based on the hypothesis that heparin regulates vimentin in the tumor-endothelium interaction and the finding of endothelial 2-DE experiment that vimentin expression altered greatly by low molecular weight heparin, we demonstrated that vimentin partly plays a role in the anti-metastatic effects of low molecular weight heparin ${ }^{[16]}$. In the present study, we further analyzed and confirmed the down-regulated expression of vimentin, which was also one of the proteins influenced by heparin and identified in both the PC-3M cells and lungs of mice burdened with B16-F10-luc-G5 cells, along with 14-3-3 zeta/delta. The 14-3-3 zeta/delta belongs to a family of highly conserved proteins that play important roles in a wide range of cellular processes including apoptosis, cell cycle progression, and signal transduction. 14-3-3 proteins regulate the cell cycle and prevent apoptosis by controlling the nuclear and cytoplasmic distribution of signaling molecules with which they interact. In our pharmacodynamics studies about heparin on PC-3M tumor cells, we found that heparin have obviously inhibitory effects on tumor growth, proliferation and cell cycle. Additionally, the 14-3-3 dimer can bind to vimentin polypeptides and regulate Raf activation by EGF ${ }^{[17]}$, 14-3-3 protein plays an organizing role in the intermediate filament network ${ }^{[18]}$, and its overexpression has been found in multiple cancers. 14-3-3 zeta and delta both have been identified as biomarkers for poor prognosis, therapy and chemoresistance in multiple tumor types ${ }^{[19,20]}$. Thus, vimentin and 14-3-3 are of interest as their protein expression levels were found to be significantly decreased by heparin in PC-3M cells. Our results of vimentin-targetted siRNA showed that partial knockdown of vimentin in PC-3M could reduce the ability of PC-3M cells proliferation. The inhibitory effects of heparin on structural organization of vimentin play an important role in its anti-tumor and anti-metastasis function. Meaningfully, activation of an Akt-14-3-3 zeta signaling pathway in promoting a multidrug-resistant phenotype has been associated with vimentin-dependent invasive behavior in diffuse large B-cell lymphoma cells ${ }^{[21]}$. These proteins may account for the effect of heparin as a chemotherapy sensitizer, but further research on the role of 14-3-3 and vimentin in multi-drug resistance to anti-tumor agents should be undertaken.

In recent years, the cytoskeleton protein vimentin has gained attention for its roles in cell migration, adhesion, EMT, and signal transduction. In cancer-related studies, vimentin 
has been found to be mainly involved in tumor mobility ${ }^{[22,23]}$. Vimentin has been shown to co-localize with $\alpha_{v} \beta_{3}$-integrin (focal contacts) ${ }^{[24]}$. Moreover, through integrin-linked kinase, integrin can regulate expression and function of vimentin. The levels of vimentin and integrin play a key role in tissue homeostasis as well, and their hyperexpression leads to tumorigenesis $^{[25,26]}$. We detected and observed that the mRNA expression levels of $\mathrm{a}_{\mathrm{v}}$-integrin and vimentin could be mediated by heparin treatment.

In conclusion, our study has demonstrated multiple effects of heparin on tumors, consistent with previous studies that have identified heparin as an anti-cancer drug with diverse targets. These effects of heparin involve the inhibition of 14-3-3 zeta/delta, vimentin and $a_{v}$-integrin expression.

\section{Acknowledgements}

This work was supported by the National Natural Science Foundation of China (No 81020108031, 30572202, 30973558, 30772571, 30901815, 30901803), the Major Specialized Research Fund from the Ministry of Science and Technology in China (No 2009ZX09103-144), Research Fund from Ministry of Education of China (111 Projects No B07001) and Scientific Research Foundation for the Returned Overseas Chinese Scholars, State Education Ministry.

\section{Author contribution}

Yan PAN, Hong ZHOU, and Xue-jun LI designed research; Yan PAN, Li-jun ZHONG, Kui CHEN, Hao-peng YANG, Yilixiati XIAOKAITI, Aikebaier MAIMAITI, and Ling JIANG performed research; Yan PAN, Xin WANG, and Li-jun ZHONG analyzed data; Yan PAN and Xue-jun LI wrote the paper.

\section{References}

1 Mandal AK, Lyden TW, Saklayen MG. Heparin lowers blood pressure biological and clinical perspectives. Kidney Int 1995; 47: 1017-24.

2 Neison RM, Cecconi O, Roberts G, Aruffo A, Linhard RJ, Bcvilaqua MP. Heparin oligosaccharides bind $L$ - and P-selection and inhibit acute inflammation. Blood 1993; 82: 3253-61.

3 Li HL, Ye KH, Zhang HW, Luo YR, Ren XD, Xiong AH, et al. Effect of heparin on apoptosis in human nasopharyngeal carcinoma CNE2 cells. Cell Res 2001; 4: 311-5.

4 Lebeau B, Chastang C, Brechot JM, Capron F, Dautzenberg B, Delaisements $\mathrm{C}$, et al. Subcutaneous heparin treatment increases survival in small cell lung cancer. Cancer 1994; 1: 38-45.

5 Altinbas M, Coskun HS, Er O, Ozkan M, Eser B, Unal A, et al. A randomized clinical trial of combination chemotherapy with and without low-molecular-weight heparin in small cell lung cancer. J Thromb Haemost 2004; 8: 1266-71.

6 Niers TM, Klerk CP, DiNisio M, Van Noorden CJ, Büller HR, Reitsma $\mathrm{PH}$, et al. Mechanisms of heparin induced anti-cancer activity in experimental cancer models. Crit Rev Oncol Hematol 2007; 3: 195207.

7 Pan Y, Iwata F, Wang D, Muraguchi M, Ooga K, Ohmoto Y, et al. Identification of aquaporin- 5 and lipid rafts in human resting saliva and their release into cevimeline-stimulated saliva. Biochim Biophys Acta 2009; 1: 49-56.
8 Boeryd B. Effect of heparin and plasminogen inhibitor (EACA) in brief and prolonged treatment on intravenously injected tumor cells. Acta Pathol Microbiol Scand 1966; 68: 347-54.

9 Hagmar B, Boeryd B. Disseminating effect of heparin on experimental tumor metastases. Pathol Eur 1969; 4: 274-82.

10 Maat B. Extrapulmonary colony formation after intravenous injection of tumor cells into heparin-treated animals. Br J Cancer 1978; 3: 369-76.

11 Ramakrishnan S, Grebe R, Singh M, Schmid-Schönbein H. Evaluation of hemorheological risk factor profile in plasmacytoma patients. Clin Hemorheol Microcirc 1999; 20: 11-9.

12 Rickles FR, Falanga A. Molecular basis for the relationship between thrombosis and cancer. Thromb Res 2001; 6: V215-24.

13 Mousa SA, Petersen LJ. Anti-cancer properties of low-molecularweight heparin: preclinical evidence. Thromb Haemost 2009; 102: 258-67.

14 Casu B, Vlodavsky I, Sanderson RD. Non-anticoagulant heparins and inhibition of cancer. Pathophysiol Haemost Thromb 2008; 36: 195203.

15 Erduran E, Tekelioglu Y, Gedik Y, Bektaş I, Hacisalihoglu S. In vitro determination of the apoptotic effect of heparin on lymphoblasts using DNA analysis and measurements of Fas and $\mathrm{Bcl}-2$ proteins by flow cytometry. Pediatr Hematol Oncol 2004; 5: 383-91.

16 Pan Y, Lei TL, Teng B, Liu JH, Zhang JZ, An Y, et al. Role of vimentin in inhibitory effects of low molecular weight heparin on PC-3M adhesion to and migration through endothelium. J Pharmacol Exp Ther 2011; 339: 82-92.

17 Tzivion G, Luo ZJ, Avruch J. Calyculin A-induced vimentin phosphorylation sequesters 14-3-3 and displaces other 14-3-3 partners in vivo. J Biol Chem 2000; 275: 29772-8.

18 Satoh J, Yamamura T, Arima K. The 14-3-3 protein epsilon isoform expressed in reactive astrocytes in demyelinating lesions of multiple sclerosis binds to vimentin and glial fibrillary acidic protein in cultured human astrocytes. Am J Pathol 2004; 165: 577-92.

19 Yang X, Cao W, Zhang L, Zhang W, Zhang X, Lin H. Targeting 14-33zeta in cancer therapy. Cancer Gene Ther 2012; 19: 153-9.

20 Neal CL, Yu D. 14-3-33 as a prognostic marker and therapeutic target for cancer. Expert Opin Ther Targets 2010; 14: 1343-54

21 Maxwell SA, Cherry EM, Bayless KJ. Akt, 14-3-3ろ, and vimentin mediate a drug-resistant invasive phenotype in diffuse large B-cell lymphoma. Leuk Lymphoma 2011; 52: 849-64.

22 Shirahata A, Sakata M, Sakuraba K, Goto T, Mizukami H, Saito M, et al. Vimentin methylation as a marker for advanced colorectal carcinoma. Anticancer Res 2009; 29: 279-81.

23 Vora HH, Patel NA, Rajvik KN, Mehta SV, Brahmbhatt BV, Shah MJ, et al. Cytokeratin and vimentin expression in breast cancer. Int J Biol Markers 2009; 23: 38-46.

24 Tsuruta D, Jones JC. The vimentin cytoskeleton regulates focal contact size and adhesion of endothelial cells subjected to shear stress. J Cell Sci 2003; 116: 4977-84.

25 Zhang X, Fournier MV, Ware JL, Bissell MJ, Yacoub A, Zehner ZE. Inhibition of vimentin or beta1 integrin reverts morphology of prostate tumor cells grown in laminin-rich extracellular matrix gels and reduces tumor growth in vivo. Mol Cancer Ther 2009; 3: 499-508.

26 Bhattacharya R, Gonzalez AM, Debiase PJ, Trejo HE, Goldman RD, Flitney FW, et al. Recruitment of vimentin to the cell surface by beta3 integrin and plectin mediates adhesion strength. J Cell Sci 2009; 122: $1390-400$. 